University of Nebraska - Lincoln

DigitalCommons@University of Nebraska - Lincoln

$1-1-2003$

\title{
Comparison of the theoretical and experimental band structure of poly(vinylidene fluoride) crystal
}

\author{
Chun-gang Duan \\ University of Nebraska at Omaha, cgduan@clpm.ecnu.edu.cn \\ Wai-Ning Mei \\ University of Nebraska at Omaha, physmei@unomaha.edu \\ J.R. Hardy \\ University of Nebraska - Lincoln \\ Stephen Ducharme \\ University of Nebraska - Lincoln, sducharme1@unl.edu \\ Jaewu Choi \\ University of Nebraska-Lincoln, jchoi@ece.eng.wayne.edu
}

See next page for additional authors

Follow this and additional works at: https://digitalcommons.unl.edu/physicsducharme

Part of the Physics Commons

Duan, Chun-gang; Mei, Wai-Ning; Hardy, J.R.; Ducharme, Stephen; Choi, Jaewu; and Dowben, Peter A., "Comparison of the theoretical and experimental band structure of poly(vinylidene fluoride) crystal" (2003). Stephen Ducharme Publications. 10.

https://digitalcommons.unl.edu/physicsducharme/10

This Article is brought to you for free and open access by the Research Papers in Physics and Astronomy at DigitalCommons@University of Nebraska - Lincoln. It has been accepted for inclusion in Stephen Ducharme Publications by an authorized administrator of DigitalCommons@University of Nebraska - Lincoln. 


\section{Authors}

Chun-gang Duan, Wai-Ning Mei, J.R. Hardy, Stephen Ducharme, Jaewu Choi, and Peter A. Dowben 
Europhys. Lett., 61 (1), pp. 81-87 (2003)

\title{
Comparison of the theoretical and experimental band structure of poly(vinylidene fluoride) crystal
}

\author{
Chun-gang Duan $\left.^{1}{ }^{*}\right)$, W. N. Mei ${ }^{1}$, J. R. Hardy ${ }^{2}$, S. Ducharme ${ }^{2}$, \\ JAEWu CHOI $^{2}$ and P. A. Dowben ${ }^{2}$ \\ 1 Department of Physics, University of Nebraska at Omaha \\ Omaha, NE 68182-0266, USA \\ 2 Department of Physics and Center for Materials Research and Analysis \\ University of Nebraska at Lincoln - Lincoln, NE 68588, USA
}

(received 21 August 2002; accepted in final form 7 October 2002)

PACS. 71.20.Rv - Polymers and organic compounds.

PACS. 71.20.-b - Electron density of states and band structure of crystalline solids.

PACS. 71.15. Ap - Basis sets (plane-wave, APS, LCAO, etc.) and related methodology (scattering methods, ASA, linearized methods).

\begin{abstract}
The electronic structure and band symmetries of the ferroelectric phase of poly (vinylidene fluoride) (PVDF) are studied by using the first-principles density-functional method. Our calculated results agree well with the angle-resolved photoemission experiments. We find that the PVDF crystal has an energy gap of about $6 \mathrm{eV}$ at the $\Gamma$-point. Large dispersion of the valence band is found only along the chain direction, which is consistent with the quasione-dimensional nature of PVDF. In particular, the band symmetries of the valence states deduced theoretically are in good agreement with experiment. Finally, further investigations on the electronic structure of poly(trifluoroethylene) (PTrFE) reveal that the replacement of $-\left(\mathrm{CH}_{2}-\mathrm{CF}_{2}\right)$ - by - $\left(\mathrm{CHF}-\mathrm{CF}_{2}\right)$ - does not change major features of the band structure.
\end{abstract}

Poly(vinylidene fluoride) $\left[\mathrm{PVDF},-\left(\mathrm{CH}_{2}-\mathrm{CF}_{2}\right)_{n^{-}}\right]$is one of the first known crystalline ferroelectric polymers. Extensive experimental and theoretical efforts have been devoted to the study of their unique piezoelectric, pyroelectric, ferroelectric, and nonlinear optical properties [1-7]. Particularly, ultrathin PVDF and its copolymer films with $30 \%$ trifluoroethylene [TrFE, - $\left.\left(\mathrm{CHF}-\mathrm{CF}_{2}\right)-\right]$ were observed to show a two-dimensional ferroelectric phase transition [8], and also exhibit novel dielectric resonance effects and have large potential application as nonvolatile polymer ferroelectric memories [9]. Thorough photoemission and inverse photoemission studies have been carried out on the high-quality copolymer films, providing an abundance of information concerning the electronic structure, band dispersions and band symmetries $[10,11]$. However, until now there has been little progress in theoretical efforts to detail and understand the electronic band structure data. The purpose of our work is: first to study the lattice structure and symmetry of PVDF from first principles, then to calculate the electronic structure, which is expected to have strong influence on material properties. In this

$\left(^{*}\right)$ E-mail: dcg@cosmos.unomaha.edu.

(C) EDP Sciences 


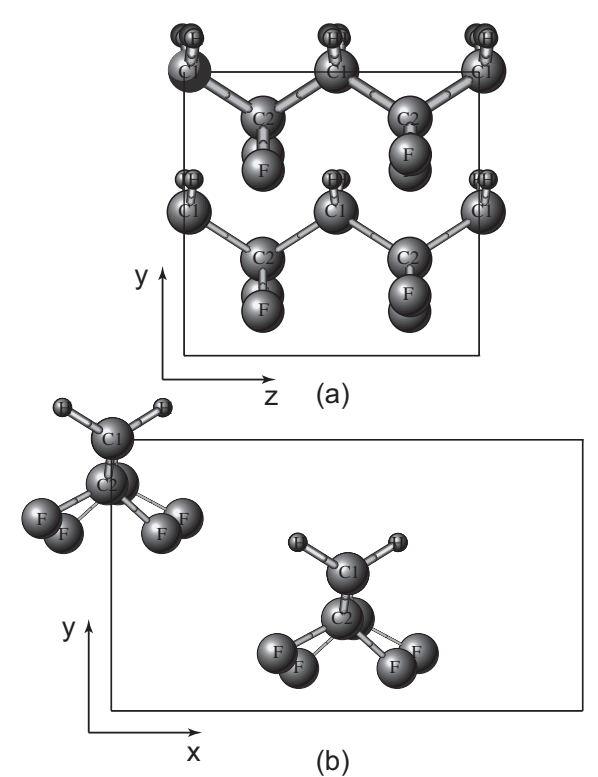

Fig. 1 - Schematic view of PVDF cell in the ferroelectric phase: (a) view along the $x$-axis; (b) view along the $z$-axis (the chain direction). The dipole direction is along the $y$-axis.

paper, we report our first-principles energy band calculations on the electronic structure of the ferroelectric phase (polar $\beta$ phase, form I) of PVDF and provide detailed understanding of the photoemission data.

The molecular conformation of the pure $\beta$ phase of PVDF is all-trans planar zigzag, with all chain dipoles oriented along the twofold axis (fig. 1). This structure is simpler than other phases of PVDF, which often involve combinations of gauche and trans conformations. Most samples used in experiment are polymorphous and contain multiple phases and incompletely oriented crystallites. This makes the accurate determination of the crystal structure of PVDF, especially the atomic coordinates, very difficult. Lando et al., based on their X-ray and NMR analyses, proposed that the unit cell of PVDF form I is orthorhombic with the space group $C m 2 m\left(C_{2 v}^{14}\right)[1]$. Later, Hasegawa et al. pointed out that there exist small $\left(\sim 7^{\circ}\right)$ dihedral deflections (fig. 1b) of the PVDF chains caused by the steric hindrance between the fluorine atoms along the chains [3]. In this work we take this latter feature into account. By choosing the fiber backbone as the $\boldsymbol{c}$-axis, the lattice constants adopted in our calculations are $a=8.58 \AA, b=4.91 \AA$, and $c=5.12 \AA$. Note that here $c$ is twice as much as the usual experimental value $2.56 \AA$, due to the dihedral deflections. From doing so, the space group is no longer $C m 2 m$ [12]; instead there are two other possible choices, namely base-centered $C c 2 m\left(C_{2 v}^{16}\right)$ or body-centered $I c 2 m\left(C_{2 v}^{22}\right)$. They differ by a displacement of $c / 2$ between neighboring chains. We then carried out calculations on both space groups with the atomic coordinates obtained from fitting the bond lengths and bond angles between $\mathrm{C}, \mathrm{H}$ and $\mathrm{F}$ atoms, which were proposed by Karasawa and Goddard according to their work of structural optimization on PVDF [4].

The first-principles band structure approach applied in this work is the full-potential linearaugmented-plane-wave method (FLAPW) [13]. No shape approximations are made to the charge densities or potentials in this all-electron method. The exchange-correlation potential is treated in the generalized gradient approximation (GGA) [14] of density-functional theory. 


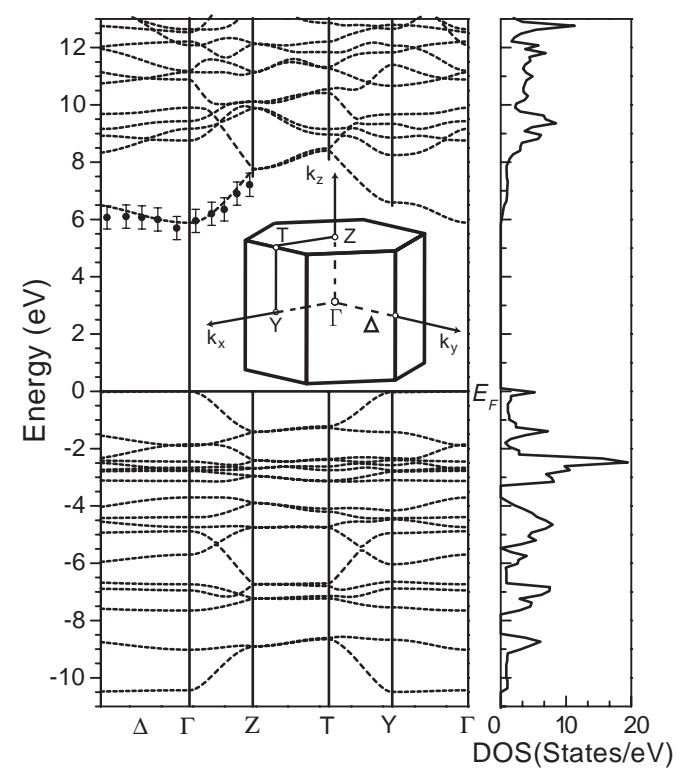

Fig. 2 - Band structure and DOS of PVDF. The inset is the Brillouin zone of the orthorhombic PVDF lattice. Solid circles indicate experimental results of band dispersions of the lowest unoccupied molecular-orbital states of PVDF copolymers.

The muffin-tin (MT) radii of $\mathrm{C}, \mathrm{H}$ and $\mathrm{F}$ atoms are chosen as 1.1, 0.7 and 1.4 a.u., respectively. The energy cut-off for plane-wave expansions in the interstitial region is $18 \mathrm{Ry}$. The upper limit of the angular momentum, $l_{\max }=10$, is adopted in the spherical-harmonic expansion of the Kohn-Sham functions inside the atom spheres. Eight $k$-points in the irreducible part of the Brillouin zone are used in the self-consistent calculation. The convergence obtained is up to $0.2 \mathrm{meV}$ of the total energy. The evaluation of the density of states (DOS) is made on a fine $k$-point grids (180 $k$-points).

At first, total energy calculations are undertaken with two candidate PVDF structures. We find that the total energy of the structure with space group $C c 2 m$ is consistently lower than that of the $I c 2 m$ structure when we increase $k$-points from 8 to 20, then 40 . Finally, we obtain a small energy difference (about $20 \mathrm{meV}$ per unit cell). Thus we choose $C c 2 m$ as the space group of the ferroelectric phase of PVDF. The band structure along high-symmetry directions in the Brillouin zone is shown in fig. 2. All energy values are taken with respect to the Fermi level. The calculated energy gap is $5.9 \mathrm{eV}$ at $\Gamma$, and compares well with the experimental value of $6.5 \mathrm{eV}$ [10]. Experimentally, the material appears to be $n$-type rather than $p$-type, most likely as a consequence of a very dilute number of charge defects in this excellent dielectric.

As we can see from fig. 2, the energy dispersion of the valence band is very small along $\boldsymbol{a}$ and $\boldsymbol{b}$ axes, which are perpendicular to the PVDF chain (denoted as $\boldsymbol{k}_{\perp}$ ). Especially the highest occupied molecular orbital is almost dispersionless along the $\boldsymbol{k}_{\perp}$ direction. On the contrary, large dispersion can be seen along the $\boldsymbol{c}$-axis, which is the PVDF chain direction (denoted as $\boldsymbol{k}_{\|}$). A similar situation is found for the lowest unoccupied band, i.e., the band dispersions along $\boldsymbol{k}_{\|}$are much larger than those along $\boldsymbol{k}_{\perp}$. This supports the findings of the angle-resolved inverse photoemission experiment $[15,16]$, which provides the experimental dispersion relationship $E(\boldsymbol{k})$ for the unoccupied bands. Thus, both experiment and theory 


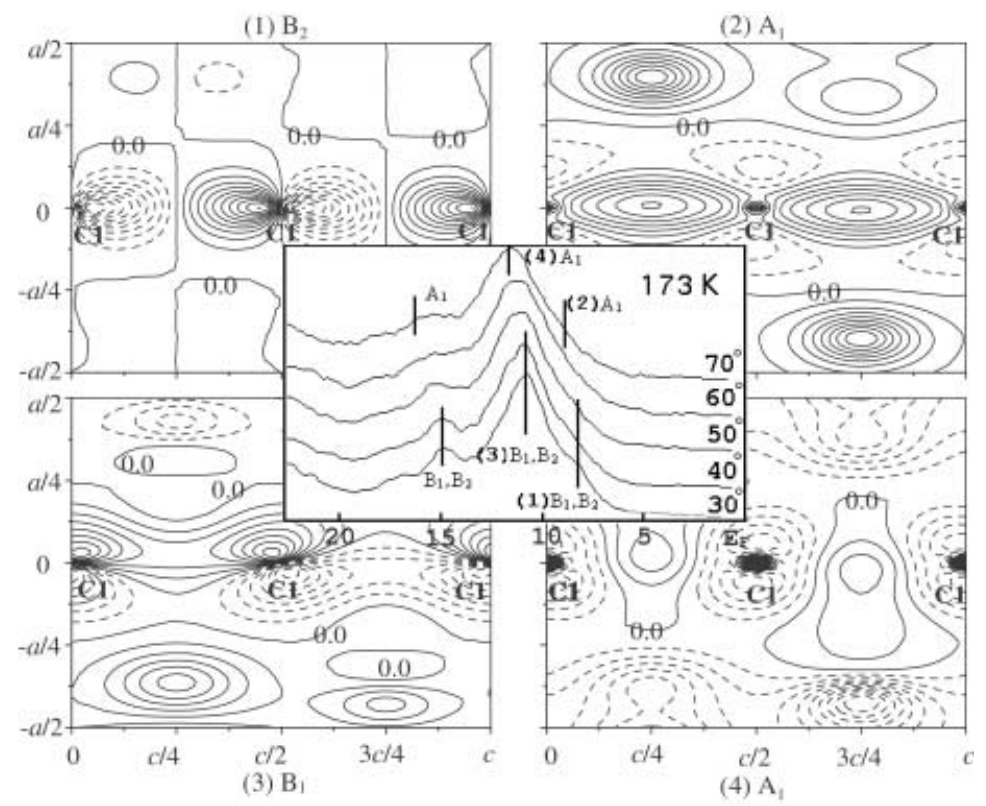

Fig. 3 - [010] contour plots of $\Gamma$-point wave functions (real part). Solid and dashed lines represent positive and negative values, respectively. Notice that two group operations $\left(I C_{2}\right)$ involve glide operation $(0,0,1 / 2)$ along the $z$-axis. Angle-resolved photoemission spectra is shown in the center panel, the abscissa is the binding energy in $\mathrm{eV}$. The light incidence angle is indicated and the photoelectron collected along the surface normal.

demonstrate that the interactions between different PVDF chains are rather weak and verify the low-dimensional electronic properties of PVDF. Furthermore, we can see from the band structure plot that our results agree quantitatively with experiment [15]. The theoretical amplitudes of the band dispersions along $\boldsymbol{k}_{\|}$and $\boldsymbol{k}_{\perp}$ are 1.6 and $0.5 \mathrm{eV}$, which agree very well with the experimental values 1.5 and $0.2 \mathrm{eV}$, respectively [16]. Figure 2 also shows the calculated DOS of PVDF, which is also in qualitative agreement with experiment [10]. Detailed analysis reveals that the top parts of the valence bands are a strong mixture of the atomic states of $\mathrm{C}, \mathrm{H}$ and $\mathrm{F}$ atoms, though $\mathrm{F} 2 p$ electron states are dominant. There exists a short terrace extending about $2 \mathrm{eV}$ at the lower end of the conduction bands, which mainly consists of $2 p$ electron states of $\mathrm{C}$ atoms, especially those bonded with $\mathrm{F}$ atoms (noted as $\mathrm{C}_{\mathrm{F}}$ ). In spite of same sizes, the MT spheres of $\mathrm{C}_{\mathrm{F}}$ contain less charge than those of $\mathrm{C}_{\mathrm{H}}$, which is caused by possessing different amounts of $2 p$ electrons, according to partial charge analysis. In addition, a small but noticeable amount of $d$ electrons are also found inside the $\mathrm{C}_{\mathrm{F}} \mathrm{MT}$ spheres. This suggests that the C-F bonding is not a simple $s$ - $p$ hybridization.

It was found from the angle-resolved photoemission data [17] that the symmetry of the occupied valence bands remains the same across the surface ferroelectric phase transition near $290 \mathrm{~K}$. Whereas the conduction band structure changes due to the surface dipole reorientation and surface Brillouin zone doubling across the phase transition, and thus strongly supports a first-order surface transition with a uniaxial polarization direction in the crystalline polymer films. Therefore, exploring both experimentally and theoretically the symmetries of PVDF electronic states should lead to further understanding of the mechanism of phase transitions in this material. In the center panel of fig. 3 , we present the valence band photoemission spectra 
TABLE I - Comparison of the binding energies and symmetries of the valence electron states at the $\Gamma$-point between theory and experiment. In order to compare with experimental results, a theoretical gap of $5.9 \mathrm{eV}$ is added to the calculated values.

\begin{tabular}{|c|c|c|c|c|c|}
\hline \multicolumn{4}{|c|}{ Binding Energy $(\mathrm{eV})$} & \multicolumn{2}{|c|}{ Symmetry } \\
\hline Theory & Exp. & & eory) & Theory & Exp. \\
\hline 5.90 & 8.2 & 2.30 & & $\mathrm{~B}_{2}$ & $\mathrm{~B}_{1}, \mathrm{~B}_{2}$ \\
\hline 7.75 & 9.0 & & 1.25 & $\mathrm{~A}_{1}$ & $\mathrm{~A}_{1}$ \\
\hline 7.80 & Forbidden & & & $\mathrm{A}_{2}$ & \\
\hline 8.64 & 10.9 & 2.26 & & $\mathrm{~B}_{1}$ & $\mathrm{~B}_{1}, \mathrm{~B}_{2}$ \\
\hline 10.29 & - & - & & $\mathrm{B}_{2}$ & - \\
\hline 10.64 & 11.8 & & 1.16 & $A_{1}$ & $A_{1}$ \\
\hline 12.84 & 14.95 & 2.11 & & $\mathrm{~B}_{1}$ & $\mathrm{~B}_{1}, \mathrm{~B}_{2}$ \\
\hline 14.91 & 16.3 & & 1.39 & $A_{1}$ & $\mathrm{~A}_{1}$ \\
\hline
\end{tabular}

of copolymer films with different light incidence angles at $173 \mathrm{~K}$. Notice that in the figure, there are significant changes in the photoemission spectra with polarization of the incident light from predominantly $p$-polarized light $\left(70^{\circ}\right)$ to predominantly $s$-polarized light $\left(30^{\circ}\right)$. The films are well ordered and crystalline with the polymer chains in the film plane [16], hence the spectra can be used to assign the symmetry of the occupied (valence) bands.

Generally speaking, in the photoemission experiments, the wave functions of the photoelectron at point $\boldsymbol{r}$ with final momentum $\boldsymbol{k}_{\mathrm{f}}$ is given as [18]

$$
\psi_{\boldsymbol{k}_{\mathrm{f}}} \propto \frac{e^{i k_{\mathrm{f}} r}}{r} \boldsymbol{A} \cdot \boldsymbol{M}^{(i)}\left(\boldsymbol{k}_{\mathrm{f}} ; \lambda\right)
$$

where $\boldsymbol{A}$ is the vector potential of the incident electromagnetic radiation, and the dipole matrix element which governs the selection rule of the transition is

$$
\boldsymbol{M}^{(i)}\left(\boldsymbol{k}_{\mathrm{f}} ; \lambda\right)=\int \mathrm{d}^{3} r \phi_{\boldsymbol{k}_{\mathrm{f}}}^{*}(\boldsymbol{r}) \boldsymbol{r} \phi_{\lambda}^{(i)}(\boldsymbol{r}) .
$$

In eq. $(2), \phi_{\boldsymbol{k}_{\mathrm{f}}}^{*}(\boldsymbol{r})$ is the distorted plane-wave-like final state in the crystal field $V(\boldsymbol{r})$, and $\phi_{\lambda}^{(i)}(\boldsymbol{r})$ is the localized or extended wave functions of the emitter. The index $i$ labels the irreducible representation of the group $G$ according to which $\phi_{\lambda}^{(i)}(\boldsymbol{r})$ transforms, while $\lambda$ labels the partner functions in the case that $i$ is a multidimensional representation. With the $C_{2 v}$ symmetry of PVDF and defining $z$ along the surface normal and perpendicular to the chain, it is known that there are four one-dimensional irreducible representations, namely $\mathrm{A}_{1}\left(s, p_{z}, d_{3 z^{2}-r^{2}}\right), \mathrm{A}_{2}\left(d_{x y}\right), \mathrm{B}_{1}\left(p_{x}, d_{x z}\right)$ and $\mathrm{B}_{2}\left(p_{y}, d_{y z}\right)$. Each of them manifests different reflection symmetries perpendicular or parallel to the molecular axis. Experimentally, it is possible to preferentially select for the $A_{1}, B_{1}$ and $B_{2}$ symmetries by adjusting the incident light from $p$-polarization to $s$-polarization, and hence accommodate the appropriate selection rules. Since the rotation axis of $G$ is the surface normal, thus the spectra obtained by collecting photoelectrons emitted normal to the surface can be used to compare with the electronic states calculated at the $\Gamma$-point. Theoretically, the band symmetries are assigned by applying group symmetry operations to the wave functions. Thus we can obtain the $\mathrm{A}_{2}$ symmetry state, which could not be observed in experiment due to dipole selection rules [18]. In addition, it is not easy to distinguish the $\mathrm{B}_{1}$ and $\mathrm{B}_{2}$ symmetry experimentally, though in the theoretical analysis we can separate them. In order to present, explicitly, the band symmetries, we also show in fig. 3 the calculated [010] wave function contour plots of the first four detected valence 
states. In table I, we list the state binding energies calculated from first principles and those measured from the angle-resolved photoemission [17] together with their band symmetries. After close examination, we find that the symmetries of the calculated states match well with those taken from experiment, except that the $\mathrm{B}_{2}$ state with binding energy $10.29 \mathrm{eV}$ could not be resolved independently in the spectra. For the calculated energies, we noticed that the experiment-theory energy differences are symmetry dependent, i.e., about $1.27 \mathrm{eV}$ for $\mathrm{A}_{1}$ states but $2.25 \mathrm{eV}$ for $\mathrm{B}_{1}\left(\mathrm{~B}_{2}\right)$ states. Usually, a constant energy, which includes the screening and band gap correction, etc., is needed to adjust to the theoretical values before direct comparison. In this study, the experimental band gap is about $0.6 \mathrm{eV}$ higher than the theoretical value, hence adding that to the calculated values leads to good agreement. The symmetry-dependent differences between theory and experiment could well be explained by differences in final-state effects in photoemission, i.e., differences in screening. This intriguing systematic discrepancy, which appears to be related to symmetry, cannot be recovered in our present theoretical scheme as it is not clear yet how to adequately include symmetry-dependent screening and correlation effects in density-functional theory calculations.

The photoemission measurements were made on a random copolymer of vinylidene fluoride with trifluoroethylene $[\mathrm{P}(\mathrm{VDF}-\mathrm{TrFE})][10,11,15,16]$, which consists of - $\left[\left(\mathrm{CH}_{2}-\mathrm{CF}_{2}\right)_{0.70}-(\mathrm{CHF}-\right.$ $\left.\left.\mathrm{CF}_{2}\right)_{0.30}\right]_{n^{-}}$chains and is more complicated than the pure PVDF used in our calculations. In order to estimate the properties of the real copolymer system, we calculated the electronic structures of pure PTrFE [-(CHF-CF $\left.)_{n^{-}}\right]$. To simplify the problem, the structure parameters of PTrFE were obtained by substituting the monomer $-\left(\mathrm{CH}_{2}-\mathrm{CF}_{2}\right)$ - in the PVDF cell by - $\left(\mathrm{CHF}-\mathrm{CF}_{2}\right)$ - and making appropriate coordinate changes. We found that the band structure of PTrFE resembles closely that of PVDF, i.e., large dispersion only occurs along chain directions. Hence this explains partly why the experimental band structure, which was measured with the copolymer system, agreed satisfactorily with the band structure calculated for the pure PVDF. At the same time, substituting $\mathrm{H}$ for $\mathrm{F}$ did result in several noticeable changes, e.g., the $2 p$ electrons of the $\mathrm{F}$ atom not only shifted the nearby energy levels and led to change in the DOS, but also slightly distorted the upper part of valence band edge and changed the bottom of conduction band. In our calculation of PTrFE, we found that the band gap is $5.5 \mathrm{eV}$ at the end of $\Delta$ line, which is different from that of PVDF $(5.9 \mathrm{eV}$ at the $\Gamma$-point).

In conclusion, we report the first electronic-structure calculation of the ferroelectric phase of PVDF crystal, from using the first-principles FLAPW method. Not only the calculated band gap and dispersions agree well with the photoemission data, but also the band symmetries match well with experiment. To the best of our knowledge, the present level of agreement has rarely been achieved for polymer systems. From the band structure calculations and photoemission measurements, we show that the interactions between different PVDF chains are rather weak, which reflects clearly the quasi-one-dimensional nature of the system. Furthermore, we calculated the electronic structure of $\mathrm{PTrFE}$ and find that replacing the $-\left(\mathrm{CH}_{2}-\mathrm{CF}_{2}\right)$ by - $\left(\mathrm{CHF}-\mathrm{CF}_{2}\right)$ - does not change major features of the band structure. Finally, this comparison between theory and experiment enhances our understanding of the electronic structures of bulk PVDF and PTrFE and further benefits the studies of PVDF and P(VDF-TrFE) films.

$$
* * *
$$

This work was supported by the Nebraska Research Initiative, the Nebraska-EPSCOR-NSF Grant EPS-9720643 and Department of the Army Grants DAAG 55-98-1-0273 and DAAG 5599-1-0106. SD, PD and WNM are grateful for the support from the Office of Naval Research. 


\section{REFERENCES}

[1] Lando J. B., Olf H. G. and Peterlin A., J. Polym. Sci., 4 (1966) 941.

[2] Farmer B. L., Hopfinger A. J. and Lando J. B., J. Appl. Phys., 43 (1972) 4293.

[3] Hasegawa R., Takahashi Y., Chatani Y. and Tadokoro H., Polym. J., 3 (1972) 600.

[4] Karasawa N. and Goddard III W. A., Macromolecules, 25 (1992) 7268.

[5] Tashiro K., Abe Y. and Kobayashi M., Ferroelectrics, 171 (1995) 281.

[6] Nalwa H. S., Ferroelectric Polymers (Marcel Dekker, New York) 1995 pp. 895.

[7] Wang T. T., Herbert J. M. and Glass A. M., The Applications of Ferroelectric Polymers (Blackie, Glasgow) 1988.

[8] Bune A. V., Fridkin V. M., Ducharme Stephen, Blinov L. M., Palto S. P., Sorokin A. V., Yudin S. G. and Zlatkin A., Nature, 391 (1998) 874.

[9] Yamauchi N., Jpn. J. Appl. Phys., 25 (1986) 590.

[10] Choi Jaewu, Dowben P. A., Pebley Shawn, Bune A. V., Ducharme Stephen, Fridkin V. M., Palto S. P. and Petukhova N., Phys. Rev. Lett., 80 (1998) 1328.

[11] Borca C. N. et al., Phys. Rev. Lett., 83 (1999) 4562.

[12] In order to reconcile the existence of the $7^{\circ}$ deflections in the PVDF chain and the fact that the experimentally observed space group is $C m 2 m$, Hasegawa et al. proposed a statistical model, in which the deflected chain and its mirror image could occur with equal probability. To simplify the calculation, we did not adopt this model in our work.

[13] Blaha P., Schwarz K. and Luitz J., WIEN97, A Full Potential Linearized Augmented PlaneWave Package for Calculating Crystal Properties (Karlheinz Schwarz, Technische Universität Wien, Austria) 1999; ISBN 3-9501031-0-4.

[14] Perdew J. P., Burke K. and Ernzerhof M., Phys. Rev. Lett., 77 (1996) 3865.

[15] Choi Jaewu, Dowben P. A., Stephen Ducharme, Fridkin V. M., Palto S. P., Petukhova N. and Yudin S. G., Phys. Lett. A, 249 (1998) 505.

[16] Choi Jaewu et al., Phys. Rev. B, 61 (2000) 5760.

[17] Choi Jaewu, Tang S.-J., Sprunger P. T., Dowben P. A., Fridkin V. M., Sorokin A. V., Palto S. P., Petukhova N. and Yudin S. G., J. Phys. Condens. Matter, 12 (2000) 4735.

[18] Maradudin A. A. and Tong S. Y., Phys. Rev. B, 34 (1986) 3678. 